Chirurg 2014 · 85:568-569

DOI 10.1007/s00104-014-2740-z

Online publiziert: 25. Juni 2014

c) Springer-Verlag Berlin Heidelberg 2014

\title{
C.T. Germer
}

Klinik für Allgemein-, Viszeral-, Gefäß- und Kinderchirurgie, Universitätsklinikum Würzburg,

Zentrum Operative Medizin (ZOM), Würzburg

\section{Laparoskopische onkologische Chirurgie des unteren Gastrointestinaltraktes}

\section{Die Evidenzlage}

Karzinome des Kolons und des Rektums stellen in Deutschland die zweithäufigste Krebserkrankung überhaupt und gleichzeitig die häufigste Tumorart des Gastrointestinaltraktes dar. Jährlich erkranken ausweislich der Statistik des RobertKoch-Institutes ca. 35.500 Männer und 28.400 Frauen am kolorektalen Karzinom.

Trotz aller Fortschritte in der adjuvanten und neoadjuvanten Therapie des Kolon- bzw. Rektumkarzinoms sowie der Ausweitung endoskopischer Verfahren zur Behandlung von Frühkarzinomen steht unverändert die chirurgische Therapie mit radikaler Primärtumorresektion und systematischer Lymphadenektomie im Zentrum der Behandlung. Als Paradebeispiel für den hohen prognostischen Einfluss einer korrekten onkologischen Operationstechnik kann die Einführung des Konzeptes der total mesorektalen Exzision in die Chirurgie des Rektumkarzinoms durch B. Heald angeführt werden [2]. Ähnliches gilt für das von W. Hohenberger inaugurierte Konzept der mesokolischen Exzision beim Kolonkarzinom $[3,5]$.

Betrachtet man die Resultate in der Behandlung des kolorektalen Karzinoms im europäischen Vergleich, so zeigt sich in der jüngst publizierten Eurocare-5-Studie, dass die Ergebnisse in Deutschland mit einem mittleren 5-Jahres-Überleben von $62,2 \%$ für das Kolonkarzinom und von $57,9 \%$ für das Rektumkarzinom über dem europäischen Durchschnitt von 57\% bzw. 55,8\% liegen [1].

Neben der konventionell offen durchgeführten Operation stellt auch die laparoskopische (minimal-invasive) Chirurgie eine Therapieoption beim kolorektalen Karzinom dar. Auch wenn die minimal-invasiv durchgeführten kolorektalen Resektionen längere Operationszeiten bedingen, sprechen zahlreiche randomisierte Studien dafür, dass im Kurzzeitverlauf perioperative Vorteile im Vergleich zur konventionell durchgeführten Operation bei unveränderter Gesamtmorbidität und Letalität resultieren. Die systematischen Reviews und Metaanalysen zeigten äquivalente onkologische Langzeitergebnisse, vor allem beim Kolonkarzinom, sodass in der neuen Version der S3-Leitlinie zum kolorektalen Karzinom die laparoskopische Resektion des Kolon-Rektum-Karzinoms bei entsprechender Expertise und geeigneter Selektion mit dem Empfehlungsgrad A und dem Evidenzlevel 1A empfohlen wird [4]. (Die Evidenzlage der laparoskopischen Chirurgie beim Kolon- bzw. Rektumkarzinom schildern Schwenk et al. und Fürst et al. in diesem Leitthemenheft.)

Trotz dieser Empfehlung und Datenlage ist offensichtlich die Akzeptanz der laparoskopischen Chirurgie des kolorektalen Karzinoms in Deutschland nach wie vor niedrig. Ausweislich der Zahlen des statistischen Bundesamtes wurden ledig- lich knapp 11\% der kolorektalen Karzinome in der Bundesrepublik Deutschland im Jahre 2012 minimal-invasiv operiert. Die in diesem Heft publizierten Daten aus der multizentrischen Beobachtungsstudie "Qualitätssicherung Kolon-Karzinom (Primärtumor)" aus dem Zeitraum 2009 bis 2011 (Ptok et al.) zeigen einen Anteil laparoskopisch durchgeführter Eingriffe von $12-21,4 \%$ in diesem Register.

\section{》) Die Akzeptanz der minimal-invasiven Chirurgie ist nach wie vor niedrig}

Die Gründe für die Zurückhaltung gegenüber der minimal-invasiven kolorektalen Chirurgie sind vielfältig - höhere Kosten, höherer technischer Aufwand, längere Operationszeiten, höherer technischer Schwierigkeitsgrad im Vergleich zu anderen minimal-invasiven Operationen tragen sicher dazu bei. Dass die Vorteile der minimal-invasiv durchgeführten Operation bei konsequentem Einsatz des sog. Fast-Track-Konzeptes weniger zum Tragen kommen, mag ein weiterer Grund sein [6].

Das vorliegende Schwerpunktheft möchte einen aktuellen Überblick über die derzeitige Evidenzlage zur laparoskopischen Chirurgie beim Kolon- und beim Rektumkarzinom bieten. Darüber hinaus wird in dem Beitrag von Ptok et al. 
die aktuelle Ist-Situation in Deutschland aus den Daten der multizentrischen Beobachtungsstudie "Qualitätssicherung Kolon-Karzinom (Primärtumor)” dargelegt, die außerhalb kontrollierter Studien wohl die Realität ist. In dem Beitrag von $L u x$ et al. aus Erlangen, wird noch einmal besonders auf die Bedeutung der mesokolischen Exzision beim Kolonkarzinom eingegangen und die hieraus resultierenden hohen technischen Ansprüche an die Chirurgen, die es auch im Falle einer laparoskopisch durchgeführten Operation einzuhalten gilt. Es stellt sich dabei auch die Frage, ob es bei der laparoskopischen Kolonchirurgie immer gelingt, über die üblichen kleinen Minilaparotomien die Präparate ohne Einrisse so zu bergen, dass die notwendige pathologische Untersuchung als Qualitätskontrolle nicht beeinträchtigt wird.

Wir hoffen Ihnen mit diesem Schwerpunktheft zur Evidenzlage der minimalinvasiven Chirurgie des kolorektalen Karzinoms einen guten und aktuellen Überblick über die derzeitige Datenlage zu diesem Thema geben zu können.

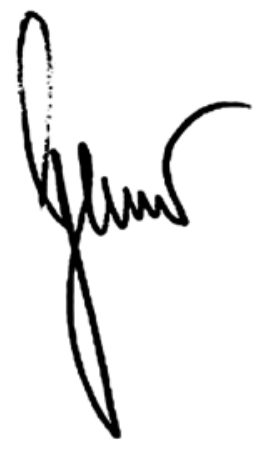

Prof. Dr. C.T. Germer

\section{Korrespondenzadresse}

Prof. Dr. C.T. Germer

Klinik für Allgemein-, Viszeral-, Gefäß- und Kinderchirurgie, Universitätsklinikum Würzburg, Zentrum Operative Medizin (ZOM), Oberdürrbacher Str. 6, 97080 Würzburg germer_c@ukw.de

\section{Einhaltung ethischer Richtlinien}

Interessenkonflikt. C.T. Germer gibt an, dass kein Interessenkonflikt besteht.

\section{Literatur}

1. De Angelis R, Sant M, Coleman MP et al (2014) Cancer survival in Europe 1999-2007 by country and age: results of EUROCARE-5 - a populationbased study. Lancet Oncol 15:23-34

2. Heald RJ, Moran BJ, Ryall RD et al (1998) Rectal cancer: the Basingstoke experience of total mesorectal excision, 1978-1997. Arch Surg 133:894899

3. Hohenberger W, Weber K, Matzel K et al (2009) Standardized surgery for colonic cancer: complete mesocolic excision and central ligation - technical notes and outcome. Colorectal Dis 11:354-364

4. Pox, Aretz S, Bischoff SC et al (2013) S3-guideline colorectal cancer version 1.0. Z Gastroenterol 51:753-854

5. Søndenaa K, Quirke P, Hohenberger W et al (2014) The rationale behind complete mesocolic excision (CME) and a central vascular ligation for colon cancer in open and laparoscopic surgery: proceedings of a consensus conference. Int J Colorectal Dis 29:419-428

6. van Bree SH1, Vlug MS, Bemelman WA et al (2011) Faster recovery of gastrointestinal transit after laparoscopy and fast-track care in patients undergoing colonic surgery. Gastroenterology 141:872880

\section{Perioperatives Management von geriatrischen Patienten}

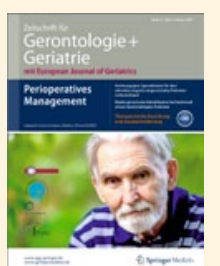

Im Vergleich zur Gesamtbevölkerung nimmt die Zahl der Notfallpatienten über 70 Jahre etwa 4-mal so schnell zu. Ähnlich verhält es sich mit der Entwicklung der

Operationszahlen. Das perioperative Management älterer Patienten gewinnt so an Bedeutung. Die Zeitschrift für Gerontologie + Geriatrie beleuchtet in Ausgabe 2/2014 dieses relativ neue Kapitel der geriatrischen Medizin.

Lesen Sie im Schwerpunktheft mehr zu folgenden Themen:

- Perioperative Risikostratifizierung geriatrischer Patienten bei nichtkardialen Eingriffen

- Algorithmus zum Management der Antikoagulation bei geriatrischen Patienten mit Hüftfraktur - „Surgeons save Blood"

- Pharmakologische Prävention des postoperativen Delirs

- Anästhesieverfahren, Mortalität und Verlauf bei geriatrischen Patienten

- Besonderheiten der perioperativen Intensivmedizin beim geriatrischen Patienten

- Perioperative Ernährungstherapie

Bestellen Sie diese Ausgabe zum Preis von 36,- EUR zzgl. Versandkosten bei Springer Customer Service Center Kundenservice Zeitschriften Haberstr. 7

69126 Heidelberg

Tel.: +49 6221-345-4303

Fax: +49 6221-345-4229

E-Mail: leserservice@springer.com

Suchen Sie noch mehr zum Thema? Mit e.Med, dem Online-Paket von Springer Medizin, können Sie schnell und komfortabel in über 600 medizinischen Fachzeitschriften recherchieren.

Weitere Infos unter: springermedizin.de/ eMed. 